\title{
Design and Analysis of Microstrip Bi-Yagi and Quad-Yagi Antenna Arrays for WLAN Applications
}

\author{
Gerald R. DeJean, Member, IEEE, Trang T. Thai, Student Member, IEEE, Symeon Nikolaou, Student Member, IEEE, \\ and Manos M. Tentzeris, Senior Member, IEEE
}

\begin{abstract}
In this letter, the design of a microstrip bi-Yagi and microstrip quad-Yagi array antenna is presented. These designs are a derivative of the original microstrip Yagi antenna array and can achieve a high gain and a high front-to-back (F/B) ratio in comparison to the conventional microstrip Yagi structure first proposed by Huang in 1989. The proposed bi-Yagi and quad-Yagi antenna arrays can also achieve a higher gain $(3-6 \mathrm{~dB})$ than the conventional microstrip Yagi array. Simple fabrication techniques can be used with these designs due to the placement of the feeding network on the same layer with the antenna elements. Furthermore, simulations and measurements demonstrate with very good agreement that the proposed arrays can achieve a gain as high as $\mathbf{1 5 . 6}$ dBi (compared to a gain of $10.7 \mathrm{dBi}$ that is achieved by the microstrip Yagi antenna array) while maintaining an F/B ratio that is relatively high.
\end{abstract}

Index Terms-Bi-/quad-Yagi array, capacitive coupling, frontto-back ratio, microstrip Yagi antenna array, quasi-endfire.

\section{INTRODUCTION}

$\mathbf{T}$ HROUGHOUT the last several years, many contributions have taken place in the design and optimization of printed microstrip Yagi antenna arrays [1]-[8]. Huang introduced the first standard design in 1989 for mobile satellite (MSAT) applications, which required a low-cost low-profile antenna that covers a $40^{\circ}$ beamwidth [1]. This design consisted of four elements of different sizes that were capacitively coupled to each other to produce a fixed beam between $20-60^{\circ}$. One of the major limitations of this design was the low front-to-back (F/B) ratio (as low as $5 \mathrm{~dB}$ ), where the back radiation considered in this design was the radiation in the elevation angles between $-90^{\circ} \leq \theta \leq 0^{\circ}$. Another limitation in the design of microstrip Yagi array antennas is the necessity of low dielectric materials $\left(\varepsilon_{r}<5\right)$ because the center-to-center spacing between elements is a function of the free space wavelength $\lambda_{0}$, not the guided wavelength $\lambda_{\mathrm{g}}$, which depends on the substrate dielectric constant. Conversely, the size of the elements is dependent on $\lambda_{\mathrm{g}}$. This means that high dielectric constant materials will result in a large center-to-center spacing between elements as their size decreases; hence, the patch elements will not be sufficiently coupled to each other. Since Huang's initial design, there have been

Manuscript received October 17, 2006; revised February 6, 2007.

G. R. DeJean was with the Georgia Institute of Technology, Atlanta, GA 30332 USA. He is now with Microsoft Research, Redmond, WA 98052 USA (e-mail: dejean@microsoft.com).

T. T. Thai, S. Nikolaou, and M. M. Tentzeris are with the Georgia Institute of Technology, Atlanta, GA 30332 USA (e-mail: gth116a@mail.gatech.edu).

Digital Object Identifier 10.1109/LAWP.2007.893104 some modifications to this antenna design process and configuration to address these limitations. Padhi and Bialkowski developed $10 \mathrm{~dB}$ and a high F/B ratio (as much as $15 \mathrm{~dB}$ ) that can be used for ISM, HIPERLAN, and millimeter-wave applications above $30 \mathrm{GHz}$ [3].

In order to meet the challenges of the design of printed microstrip Yagi antenna arrays with quasi-endfire radiation (radiation between broadside $\left(\theta=0^{\circ}\right)$ and endfire $\left(\theta=90^{\circ}\right)$ ) that can achieve a high gain $(>12 \mathrm{dBi})$ while maintaining a low cross-polarization and a high $\mathrm{F} / \mathrm{B}$ ratio, two new designs are proposed in this letter, which are derived from the microstrip Yagi array presented in [3]. The first structure is called the microstrip bi-Yagi array and the second is called the microstrip quad-Yagi array. The microstrip bi-Yagi and quad-Yagi arrays can achieve gains of 13.0 and $15.6 \mathrm{dBi}$, respectively, while a high F/B ratio is maintained. These qualities are essential to the design of planar antenna geometries that require high-gain quasi-endfire radiation patterns with $\mathrm{F} / \mathrm{B}$ ratios around $8-10 \mathrm{~dB}$ that can alleviate propagation loss effects through line-of-sight reception of waves at angles off broadside for applications such as wireless video transfer, millimeter-wave ad hoc sensor networks, and point-to-multipoint wide-band links.

\section{ANTENNA DESIGN AND PRINCIPLES OF OPERATION}

The proposed microstrip bi-Yagi and quad-Yagi array antenna designs are displayed in Figs. 1 and 2, respectively. These designs are a derivative of the original microstrip Yagi antenna array [3] in which a high gain is obtained through the constructive interference of two individual microstrip Yagi structures (R-D-D1 $1_{\mathrm{T}}-\mathrm{D} 2_{\mathrm{T}}$ and R-D-D $\left.1_{\mathrm{B}}-\mathrm{D} 2_{\mathrm{B}}\right)$ that maximally radiate at $10^{\circ} \leq \phi \leq 16^{\circ}$ and $-16^{\circ} \leq \phi \leq-10^{\circ}$, respectively. (An illustration of this antenna is shown in Fig. 3.) The operational frequency is around $5.2 \mathrm{GHz}$, but frequency scaling of this design is quite simple since the only manufacturing tolerance is the minimum trace of the metals (no via processing required). The major benefit of these structures, in comparison to the structure in [3], is the increased gain (by 3-6 dB) that can be achieved based on the design of the antenna. The common dimensions of the antennas in Figs. 1 and 2 are as follows: the length and width of the reflectors $(\mathrm{R})$ is $\left(\mathrm{L}_{\mathrm{R}}=\right) 245 \times\left(\mathrm{W}_{\mathrm{R}}=\right) 1002$ mil, the length and width of the driven element $(\mathrm{D})$ is $\left(\mathrm{L}_{\mathrm{D}}=724 \times\left(\mathrm{W}_{\mathrm{D}}=\right.\right.$ ) 724 mil, and the lengths and widths of the director 1 (D1) and director2 (D2) elements are $\left(\mathrm{L}_{\mathrm{D} 1}=\mathrm{L}_{\mathrm{D} 2}=\right) 688 \times\left(\mathrm{W}_{\mathrm{D} 1}=\right.$ $\left.\mathrm{W}_{\mathrm{D} 2}=\right) 492 \mathrm{mil}$. The distance between the elements along the $x$-axis (g) is 35 mil. These values were chosen to optimize the 


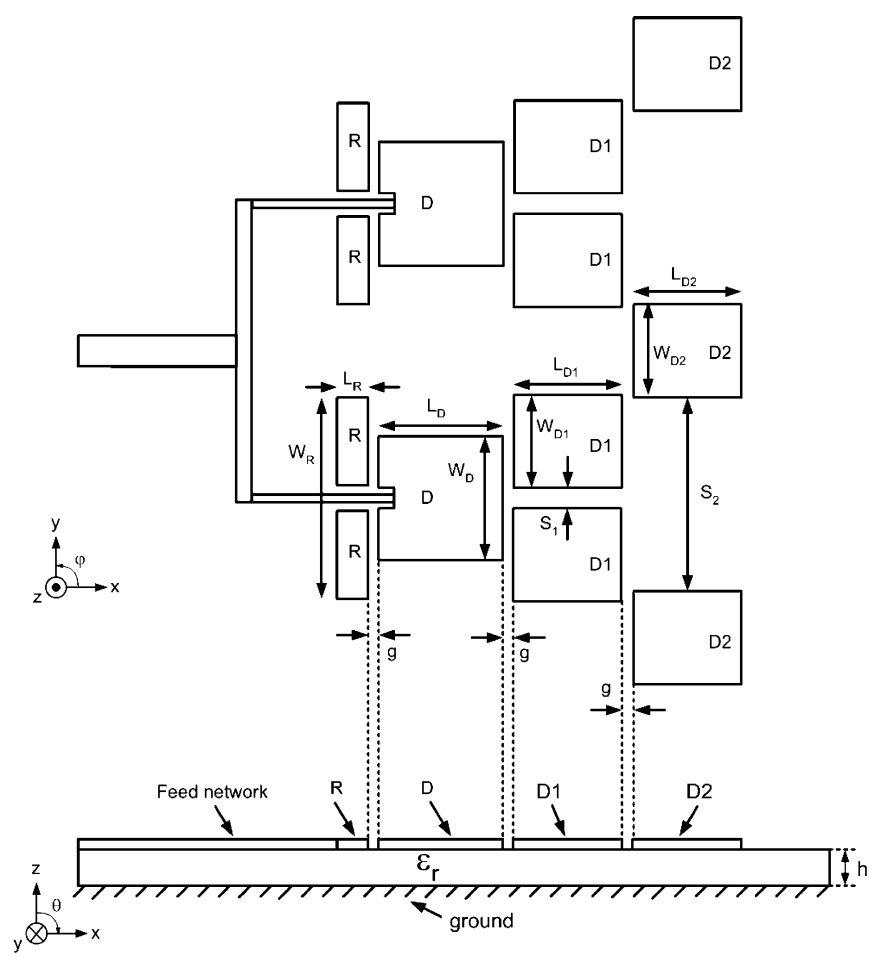

Fig. 1. Illustration of microstrip bi-Yagi array antenna.

$\mathrm{F} / \mathrm{B}$ ratio and the gain of the antenna. Furthermore, the distances between the director 1 and director 2 elements are represented by $S_{1}$ and $S_{2}$, respectively. $S_{1}$ is $72 \mathrm{mil}$ and $S_{2}$ is $902 \mathrm{mil}$. The small value of $S_{1}$ is due to the need to enhance the coupling between the driven element and the D1 elements, while conversely, the larger value for $S_{2}$ is due to the need for a wide aperture length to achieve an increased gain. These values were chosen to optimize the F/B ratio and the gain of the antenna. In the microstrip bi-Yagi array design, two conventional microstrip Yagi arrays have been connected in a composite array format where a common D2 element has been used in order to prevent H-plane sidelobes that can arise when the center-to-center separation between the elements is greater than one wavelength $(\lambda)$. Although the center-to-center spacing between the driven elements is 1548 mil $(1.5 \lambda)$, the $\mathrm{H}$-plane sidelobes are $17 \mathrm{~dB}$ below the main beam. Conversely, in the microstrip bi-Yagi array design, four conventional microstrip Yagi arrays have been connected in an array format where three common D2 elements are used to connect pairs of microstrip Yagi arrays. The center-to-center spacing between the driven elements is also $1548 \mathrm{mil}(1.5 \lambda)$. A smaller value for center-to-center spacing (1.0-1.5 $\lambda$ ) can further minimize sidelobes in the $\mathrm{H}$-plane, but the gain becomes smaller. On the other hand, larger values (greater than $2.0 \lambda$ ) can greatly increase the gain at the expense of obtaining a sidelobe level (SLL) less than $8 \mathrm{~dB}$. So, this value $(1.5 \lambda)$ is a tradeoff between achieving a minimal SLL and high gain. The size of the substrate for the microstrip bi-Yagi array is $4560 \times 5400 \mathrm{mil}$, while the quad-Yagi array has a size of $4940 \times 8496$ mil. The size of the substrate in the $y$-direction increases by $1548 \mathrm{mil}$ each time a microstrip Yagi array antenna is added to the larger array to support the antenna. Both antenna structures are designed on a double copper $(\mathrm{Cu})$ clad board of RT/duroid 5880

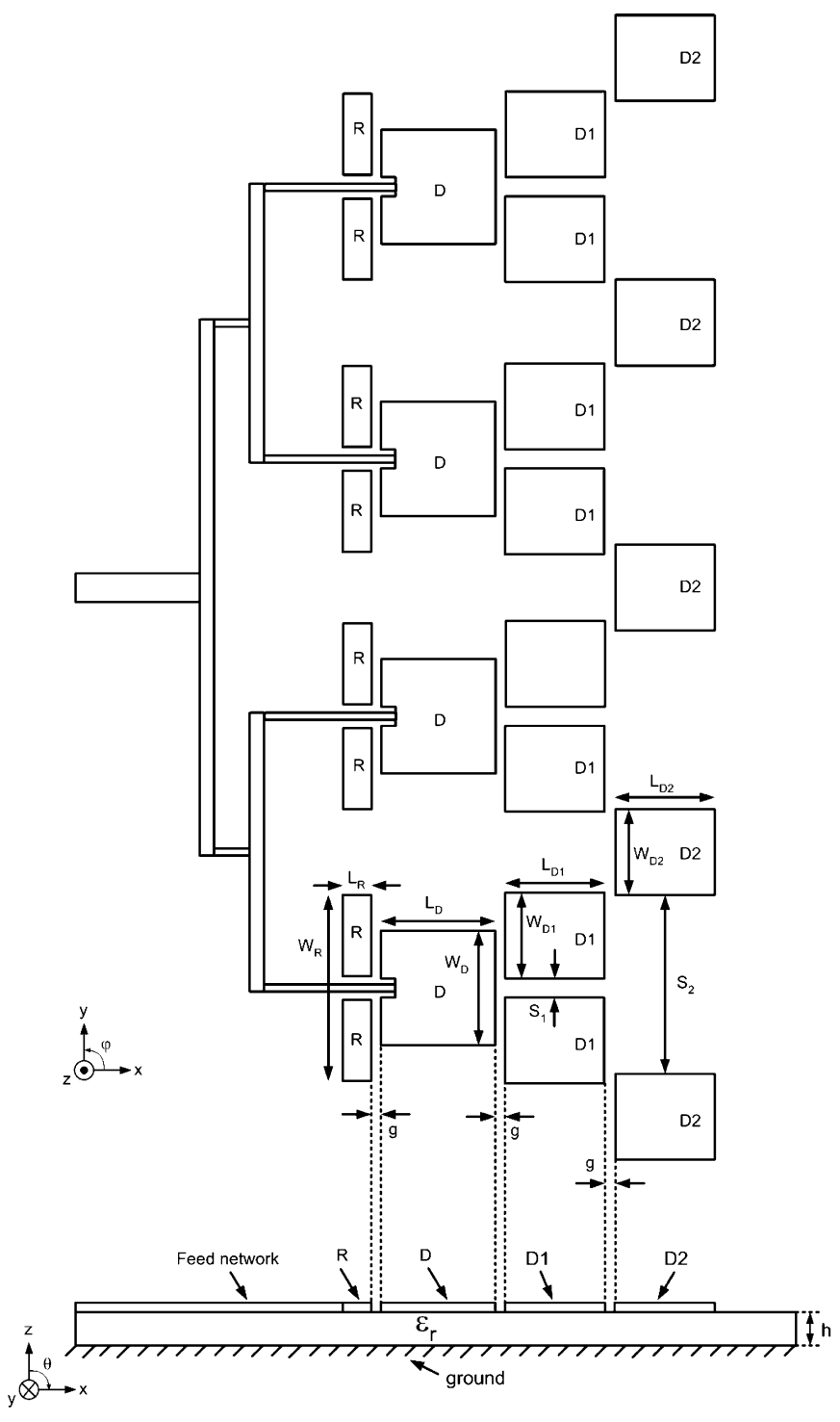

Fig. 2. Illustration of microstrip quad-Yagi array antenna.

material $\left(\varepsilon_{r}=2.2, \tan \delta=0.0009\right.$ at $\left.10 \mathrm{GHz}\right)$. The thickness of the substrate (h) is 62 mil. A thicker substrate could lead to a larger bandwidth, but the Yagi effect of quasi-endfire radiation would be degraded considerably due to the surface waves in the substrate. In order to maintain the simplicity of the fabrication on a double clad copper $(\mathrm{Cu})$ board, gaps are inserted between the reflector patches and the feedlines are connected to the driven patch through gaps in the reflectors.

The concept behind the mechanism of a printed microstrip Yagi array mirrors the idea of coupling energy to additional patches. The microstrip Yagi array is excited through the driven patch $\mathrm{D}$, and the main beam is tilted to a maximum angle between $33^{\circ}-42^{\circ}$ through capacitively coupling energy to the D1 patches. The main beam angle is mainly controlled by the gap between the driven patch and D1 elements. The high F/B ratio is obtained through the use of two D1 and two D2 patches. The D1 elements are used to establish the directionality of the beam as well as to increase the impedance bandwidth of the antenna due to the close proximity between the resonant modes of D1 and the driven element. (Note that since the resonant length of 


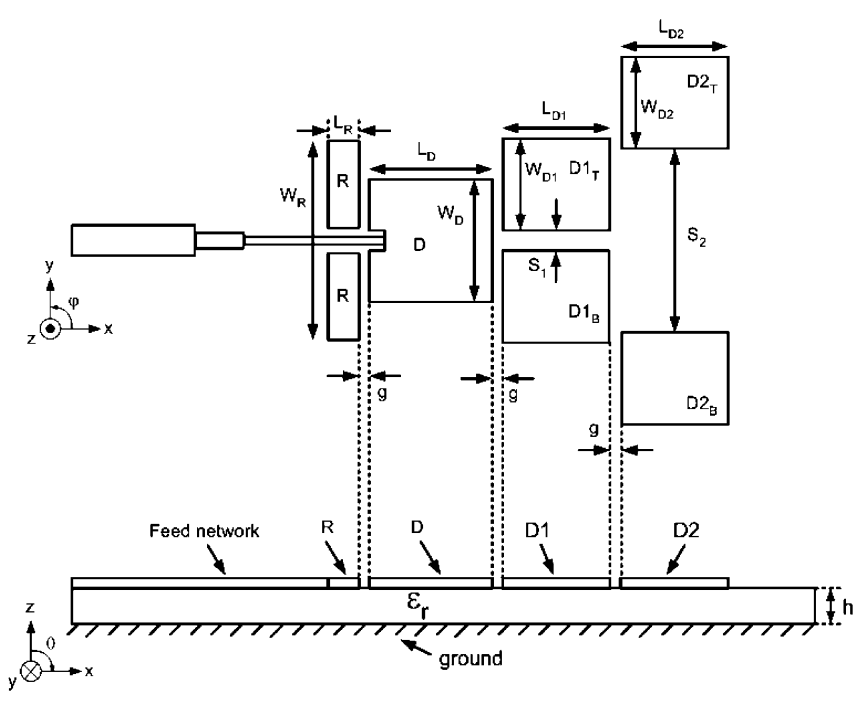

Fig. 3. Illustration of original microstrip Yagi array antenna.

D1 is slightly shorter than the driven element, around 5\%, it will resonate at a slightly higher frequency, but the combination of these modes will produce an increase in bandwidth. If the length is too small, the resonant frequency will be too high and the two modes will be too far apart to produce a wider bandwidth.) The D2 patches are used to increase the gain of the design as well as assist in steering the main beam to larger elevation angles. It is important for the value of $\mathrm{S}_{1}$ to be small $\left(<0.05 \lambda_{\text {eff }}\right.$, where $\lambda_{\text {eff }}=\mathrm{c} /\left(\mathrm{f}_{\mathrm{r}}^{*} \varepsilon_{\mathrm{eff}}^{1 / 2}\right)$ and $\varepsilon_{\mathrm{eff}}$, the effective dielectric constant lies in the range of $1<\varepsilon_{\text {eff }}<\varepsilon_{r}$ ) in order to achieve a sufficient coupling of the fields to the D1 elements. Conversely, the value of $\mathrm{S}_{2}$ has to be as large as possible (around $0.55 \lambda_{\text {eff }}$ but too large to prevent coupling enough energy from the D1 elements to the D2 elements). This is associated with the role of the D2 patches to increase the gain of the antenna by maintaining a large effective aperture length between the elements. The decrease in the backside radiation allows the effect of the reflector patch to be minimal. Through simulation, the length $L_{R}$ of the reflector is designed to be about $1 / 4$ its total width $W_{R}$. A shorter length can result in increased backside radiation. A larger length is unnecessary for reducing the backside radiation (it will only increase the size of the design, while the radiation performance will stay the same). In addition, feeding through the reflector patches has negligible effect on the impedance mismatch between the microstrip coplanar waveguide transition because the impedance difference between the lines is less than $5 \Omega$ and both lines have impedances above $100 \Omega$.

\section{Simulated AND MeAsured Results}

The microstrip bi-Yagi and quad-Yagi array antennas have been simulated using MicroStripes 7.0, a three-dimensional (3-D) full-wave simulator that solves for the electric and magnetic fields via the transmission line matrix method. After an optimized design was obtained, the two antennas were fabricated by Prototron Circuits, as shown in Fig. 4. The simulated return loss versus frequency is presented in Fig. 5 compared to that of the original (one branch) microstrip Yagi array design. The bandwidths of the three designs are as follows: microstrip

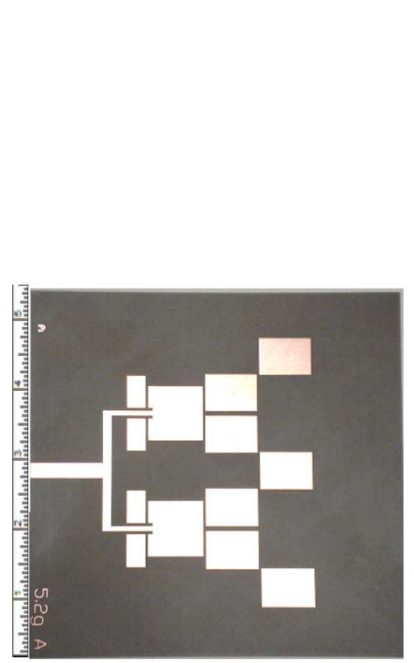

(a)

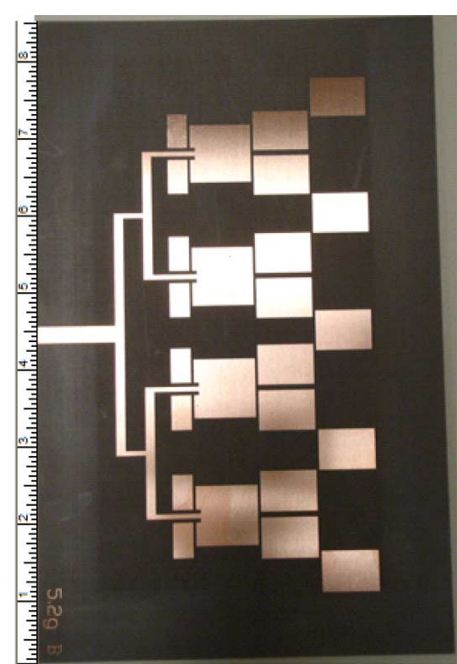

(b)
Fig. 4. Illustration of fabricated (a) microstrip bi-Yagi array and (b) microstrip quad-Yagi array antenna. The scales are in inches.

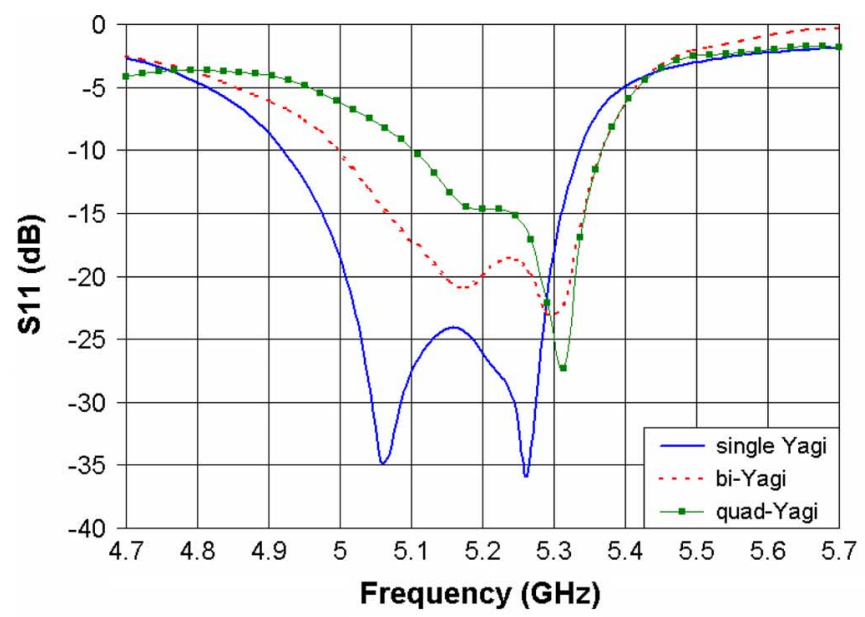

(a)

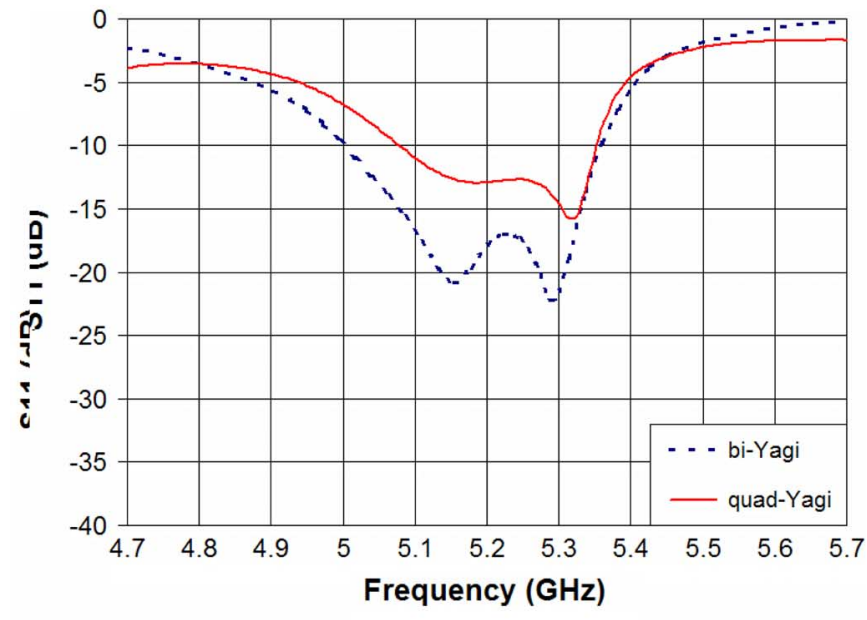

(b)

Fig. 5. (a) Simulated return loss of the three Yagi arrays and (b) measured return loss of the designs proposed in this letter.

Yagi: $8.1 \%$; bi-Yagi: 7.1\%; and quad-Yagi: 5.0\%. In comparing the three designs, it seems that the bandwidth tends to decrease 


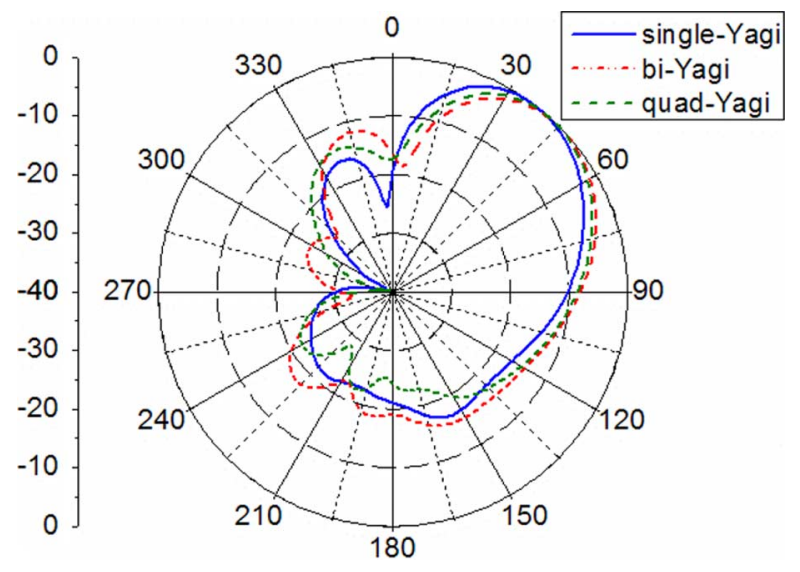

Fig. 6. Simulated 2-D radiation patterns of the three Yagi arrays at $5.2 \mathrm{GHz}$.

TABLE I

VARIATION OF GAIN AND F/B RATIO VERSUS FREQUENCY OF MiCROSTRIP BI-YAGI ARRAY

\begin{tabular}{|c|c|c|}
\hline Frequency $(\mathrm{GHz})$ & Gain $(\mathrm{dBi})$ & F/B Ratio $(\mathrm{dB})$ \\
\hline 5.1 & 12.5 & 14 \\
\hline 5.2 & 13.0 & 12.5 \\
\hline 5.3 & 13.3 & 10.7 \\
\hline
\end{tabular}

TABLE II

VARIATION OF GAIN AND F/B RATIO VERSUS FREQUENCY OF MICROSTRIP QUAD-YAGI ARRAY

\begin{tabular}{|c|c|c|}
\hline Frequency $(\mathrm{GHz})$ & Gain $(\mathrm{dBi})$ & F/B Ratio $(\mathrm{dB})$ \\
\hline 5.1 & 15.3 & 16 \\
\hline 5.2 & 15.6 & 14 \\
\hline 5.3 & 16.0 & 11.5 \\
\hline
\end{tabular}

as more microstrip Yagi arrays are added to produce a larger array. The smaller bandwidth of the quad-Yagi array may be due to the shift of the lower resonance of the driven element to a higher frequency. The measured return loss plots versus frequency of the antennas are also displayed in Fig. 5. The measured bandwidths of the bi-Yagi and quad-Yagi designs are $6.9 \%$ and $5.2 \%$, respectively. Although the lower and higher resonances of the measured designs occur at similar frequencies, the return loss of the quad-Yagi array is higher than that of the bi-Yagi array, resulting in a smaller bandwidth.

The simulated (normalized) two-dimensional (2-D) radiation patterns comparing the three Yagi designs at $5.2 \mathrm{GHz}$ are presented in Fig. 6. From this plot, it is observed that the F/B ratio tends to decrease as more Yagi arrays are included to produce the larger array. At some frequencies close to $5.2 \mathrm{GHz}$, the F/B ratio can be increased as the cost of a lower gain (by $0.5 \mathrm{~dB}$ ); hence, there is a tradeoff. Tables I and II show how the gain and F/B ratio varies with frequency. The angle of maximum radiation for all the designs is between $35^{\circ}-45^{\circ}$, while the beamwidth coverage is approximately $40^{\circ}$. Figs. 7 and 8 display the measured (normalized) 2-D radiation patterns of the microstrip bi-Yagi and quad-Yagi arrays at $5.2 \mathrm{GHz}$. A good agreement is observed between the simulated and measured results in the bi-Yagi array, although the measured design has a slightly lower $\mathrm{F} / \mathrm{B}$ ratio $(10 \mathrm{~dB})$ in comparison to simulation. The gain is $13.0 \mathrm{dBi}$ and the cross-polarization is below -25

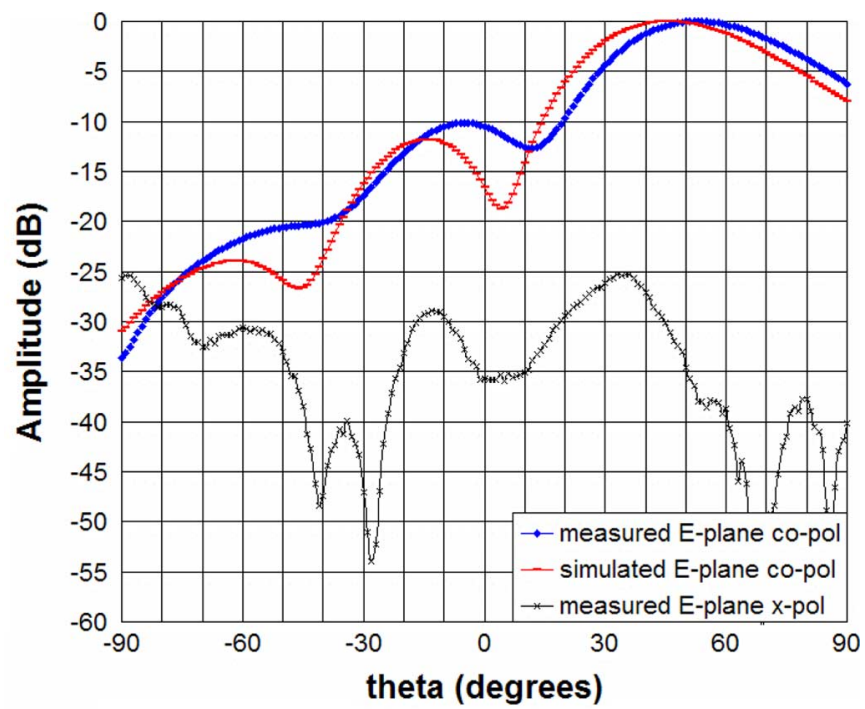

Fig. 7. Measured 2-D radiation pattern of the microstrip bi-Yagi array at $5.2 \mathrm{GHz}$.

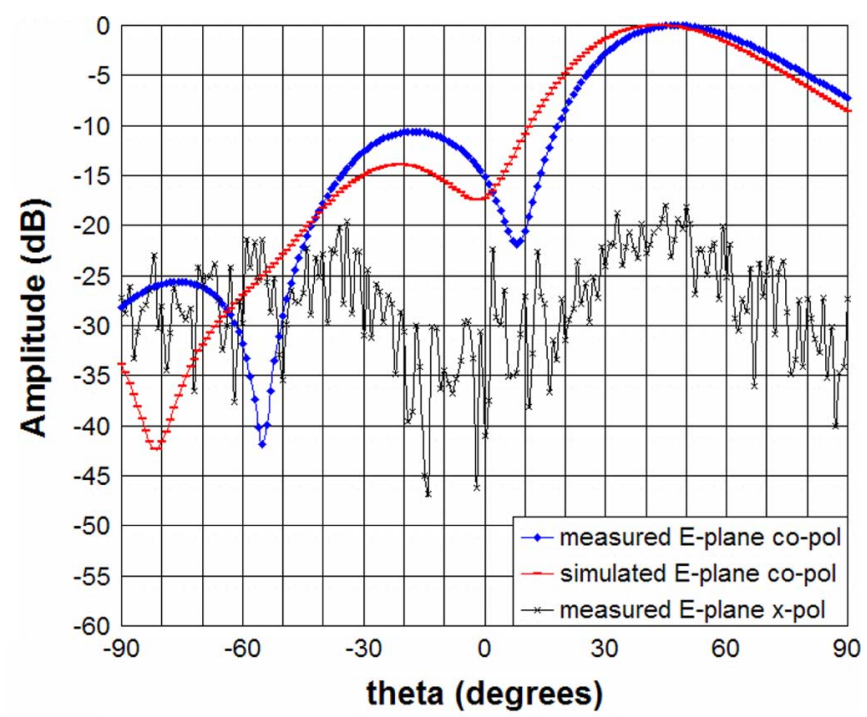

Fig. 8. Measured 2-D radiation patterns of the microstrip quad-Yagi array at $5.2 \mathrm{GHz}$.

dB. The quad-Yagi array also exhibits a good agreement between the simulations and measurements. For this design, a gain of $15.6 \mathrm{dBi}$ can be obtained with a cross-polarization below $-18 \mathrm{~dB}$. Considering that these structures use a highly conductive metal $(\mathrm{Cu})$ that was printed on a low-loss dielectric, the efficiencies of all the Yagi designs presented are greater than $89 \%$.

\section{CONCLUSION}

Two new antenna array designs based on the original microstrip Yagi array antenna (Fig. 3) have been presented: the microstrip bi-Yagi array and the microstrip quad-Yagi array. Simple fabrication techniques that are inexpensive can be employed to realize these structures due to the placement of the feeding structure on the same layer as the antenna. The measurement results of the return loss show that bandwidths close to $7 \%$ can be achieved through this design. Furthermore, the measured 
radiation pattern performance agrees well with the simulated results in terms of the beamwidth, and maximum angle of radiation for both structures and gains of 13.0 and $15.6 \mathrm{dBi}$ can be achieved with these designs with high efficiencies (above 89\%). The cross-polarization level is below $-18 \mathrm{~dB}$ and the measured $\mathrm{F} / \mathrm{B}$ ratio is around $10 \mathrm{~dB}$. Based on these results, these antenna structures could be easily integrated with 3-D modules that consist of embedded passives, filters, and monolithic microwave integrated circuits to realize a wireless system-on-package RF front end devices for ultrafast applications in wireless local-area network (WiFi, WiMax) and millimeter-wave frequencies.

\section{REFERENCES}

[1] J. Huang, "Planar microstrip Yagi array antenna," in Proc. IEEE-APS Symp., Jun. 1989, vol. 2, pp. 894-897.
[2] S. K. Padhi and M. E. Bialkowski, "Investigations of an aperture coupled microstrip Yagi antenna using PBG structure," in Proc. IEEE-APS Symp., Jun. 2002, vol. 3, pp. 752-755.

[3] G. R. DeJean and M. M. Tentzeris, "A new high-gain microstrip Yagi array antenna with a high front-to-back (F/B) ratio for WLAN and millimeter-wave applications," IEEE Trans. Antennas Propag., vol. 55, pp. 298-304, Feb. 2007.

[4] D. Gray, J. Lu, and D. Thiel, "Electronically steerable Yagi-Uda microstrip patch antenna array," IEEE Trans. Antennas Propag., vol. 46, pp. 605-608, May 1998.

[5] A. Densmore and J. Huang, "Microstrip Yagi antenna for mobile satellite service," in Proc. IEEE-APS Symp., Jun. 1991, vol. 2, pp. 616-619.

[6] J. Huang and A. Densmore, "Microstrip Yagi antenna for mobile satellite vehicle application," IEEE Trans. Antennas Propag., vol. 39, pp. 1024-1030, Jul. 1991.

[7] S. Padhi and M. Bialkowski, "Investigations of an aperture coupled microstrip Yagi antenna using PBG structure," in Proc. IEEE-APS Symp., Jun. 2002, vol. 3, pp. 752-755.

[8] S. Ke and K.-L. Wong, "Rigorous analysis of rectangular microstrip antennas with parasitic patches," in Proc. IEEE-APS Symp., Jun. 1995, vol. 2, pp. 968-971. 